\title{
Search for connections between isolated radio pulsars and supernova remnants
}

\section{Lisa Chmyreva}

Sternberg Astronomical Institute of MSU

E-mail: lisa.chmyreva@mail.ru

\section{Grigory Beskin}

Special Astrophysical Observatory, Russian Academy of Sciences

E-mail: beskinasao.ru

\section{Anton Biryukov}

Sternberg Astronomical Institute of $M S U$

E-mail: emanesai.msu.ru

\begin{abstract}
We have developed a method of searching for connections between radio pulsars and supernova remnants, based on their kinematic characteristics. We investigate fairly young radio pulsars with known proper motions and estimated distances (dispersion measures), and supernova remnants located no more than 1-2 kpc from them. Using a specified radial velocity distribution for these pulsars, we have constructed 100-200 thousand trajectories of their possible motion in the Galactic gravitational field on a time scale of a million years back. The probabilities of their close encounters with SNRs at epochs consistent with the age of the pulsar are analyzed. When these probabilities exceed considerably their reference values obtained by assuming a purely random encounter between the objects, we conclude that the pulsars may have originated in the SNRs under consideration. Out of eight pre-selected pairs of pulsar-SNR association candidates, two pairs (J1829-1751 / G16.2-2.7 and J1833-0827 / G24.7-0.6) have shown that they could be connected.
\end{abstract}

25th Texas Symposium on Relativistic Astrophysics

December 6-10, 2010

Heidelberg, Germany 


\section{Introduction}

Massive stars end their life in supernova explosions, becoming either neutron stars (pulsars) or black holes. The expansion of the shell of a supernova remnant (SNR), although fast, will eventually stop, while the formed pulsar will continue moving with a high velocity in the range of $10^{2}-10^{3} \mathrm{~km} / \mathrm{s}$ (see, e.g., [1]). In some cases, it is possible for fast pulsars to fly outside the shells of their SNRs [2]. Searching for connections between currently isolated pulsars and SNRs is of great interest, as it would allow us to localize the place of birth of the pulsar, and to estimate its real age. If the pulsar was born in a binary system, the pulsar-SNR association would allow us to find the place of disruption, as well as help locate the secondary component, if that should be a black hole. Because of the larger mass of its progenitor, the black hole would have formed first. It would not get a kick when the second massive component explodes as a supernova, hence, would not dislocate far from the place of binary disruption.

In this work, we consider fairly young isolated pulsars with known proper motions and distances, and SNRs within 1-2 kpc of their current location. We trace the pulsars' trajectories back in time, determining the distance of their approaches to the SNRs under consideration within the timescale of up to a few hundred thousand years. In cases of close approaches, it could be said that the objects may have a common origin. The consistency of the pulsar's characteristic age $\tau_{c h}$ ( $\tau_{c h}=P / 2 \dot{P}$, where $P$ is the pulsar period), the age of the SNR, and the time of approach would be an additional criterion for the validity of the hypothesis of a common origin. The significance of this hypothesis is estimated by comparing the probability of the encounter found with that for any two objects with arbitrary proper motions.

\section{The method}

Searching for the kinematic association of a pulsar and a SNR is similar to associating two pulsars (see our previous work, [3]): it consists of determining the closest approaches of their reconstructed trajectories in the past.

The main problem in studying the kinematics of pulsars is the absence of direct measurements of their radial velocities $V_{r}$. At the same time, the source of information about the latter is the distribution function of one (any) of the three peculiar velocity components of a pulsar at the time of its birth. The total velocity is assumed to be isotropic relative to the LSR. Therefore, the same function can also be used for the peculiar component of the pulsar's $V_{r}$. It is constructed from the set of data on all of the investigated pulsars as a solution of the maximum likelihood problem.

Another problem is that both the ages of pulsars and of SNRs are not known precisely. The pulsar's characteristic age may only give us a rough estimate of the real age. As for SNRs, they may be older than previously thought. The well known relations $R(t) \sim t^{2 / 5}$ for the Sedov phase and $R(t) \sim t^{2 / 7}$ for the radiative phase, may only be used if the radius $R$ is known. If the radius in not known, as would be in cases where the distance estimate of the SNR is unreliable, it may be obtained from the empirical $\Sigma-D$ relations, which again, only give a rough estimate. The SNR's age calculated from these relations also depends considerably on the density of interstellar medium. Associating pulsars and SNRs will help to solve the problem of ages, as we would have 
three separate estimates of the same epoch: the pulsar's characteristic age, the age of the SNR, and the kinematic age.

As in our previous work of searching for pulsar associations [3], we solve the equation of motion for pulsars with various initial conditions, and simulate the trajectories of their possible motion. The trajectories are traced into the past on a timescale of up to $10^{6}$ years, and the distances $\rho_{\min }$ of closest approaches to the centers of SNRs are calculated. $\tau_{c h}$ of the pulsar and the epoch of closest approach $T\left(\rho_{\min }\right)$ are then compared. A small $\rho_{\min }$ might indicate that the objects under consideration have a common origin.

There is always a chance that the encounter found is purely coincidental. The criterion that would allow us to determine the significance of the probability $P(\rho)$ for a given pair of objects to approach one another to some small distance $\rho$ in the past consists of comparing $P(\rho)$ and $P_{0}(\rho)$, where the latter is the probability for the SNR and a random, kinematically independent pulsar (with no information on its proper motion) to approach to a distance no larger than $\rho$, calculated taking into account the pulsar density in the Galaxy.

If $P(\rho)$ is too high for the simulated number of approaches to be explained only by random factors $\left(P(\rho) \gg P_{0}(\rho)\right)$, then the pair under study stands out among the others kinematically, and there is reason to suggest that they have a common origin.

\section{Results and discussion}

To find possible kinematic associations of pulsars and SNRs we first formed an initial sample of isolated radio pulsars with known distances and proper motions, and whose $\tau_{c h}$ does not exceed $10^{6}$ years. The latter is due to the relatively short lifetime of SNRs, which are detectable only up to an age of several hundred years, as well as the unreliability of the characteristic age of the pulsar; the parameters were taken mostly from the ATNF database [4] (http://www.atnf.csiro. $\mathrm{au} /$ research/pulsar/psrcat). We then selected SNRs (mostly from the Green catalog [5]), whose current separation from the pulsars under consideration does not exceed $\sim 1 \mathrm{kpc} ; 10000-15000$ trajectories were simulated for each of these pairs of objects on a timescale of $\sim 10^{6}$ years. Out of those pairs, we selected ones for which the probability density of the distribution of separations between the components had a distinct sharp peak at $\rho \leq 200 \mathrm{pc}$; for each of these pairs, a further 100000 - 200000 trajectories were then simulated. After the distributions $P(\rho), P_{0}(\rho)$, and $T(\rho)$ were constructed, the regions of the pairs' closest approaches were determined starting from $\rho$ $=5$ pc. The resulting 8 pairs are: J1835-1106 / G22.7-0.2, J1835-1106 / G18.9-1.1, J1832-0827 / G22.7-0.2, J1915+1009 / G45.7-0.4, J1833-0827 / G24.7-0.6, J1832-0827 / G23.3-0.3, J1833-0827 / G23.3-0.3, and J1829-1751 / G16.2-2.7 (see Table 1).

The best result in terms of probabilities was shown by J1829-1751 and G16.2-2.7 (see Figures 1 and 2). The probability $P(\rho \leq 5 \mathrm{pc})$ exceeds $P_{0}(\rho \leq 5 \mathrm{pc})$ by 960 times, and there is a good chance that these objects have a common origin. The epoch of closest approach for this pair is about 35000 years. The age of the SNR is roughly 24500 years, with the range of 13000-49000 years, as calculated using the relation $D(t)=(1.03 \pm 0.02) t^{0.34 \pm 0.02}$ [6]. In this case, the correlation of the SNR age and the epoch of approach is ideal. The pulsar's characteristic age, on the other hand, is 877000 years, which could mean that this is the case where $\tau_{c h}$ greatly exceeds the real age. 


\begin{tabular}{c|c|c|c|c|c|c|c|c}
\hline \hline Pulsar & SNR & $\begin{array}{c}\rho_{\min } \\
(\mathrm{pc})\end{array}$ & $\begin{array}{c}\rho_{0, \min } \\
(\mathrm{pc})\end{array}$ & $P_{0}(5)$ & $P(5)$ & $\begin{array}{c}T(5) \\
(\mathrm{yr})\end{array}$ & $\begin{array}{c}\tau_{c h} \\
(\mathrm{yr})\end{array}$ & $\begin{array}{c}t_{S N R} \\
(\mathrm{yr})\end{array}$ \\
\hline $\mathrm{J} 1829-1751$ & $\mathrm{G} 16.2-2.7$ & 2.6 & 20.7 & $1 \times 10^{-7}$ & $9.6 \times 10^{-5}$ & 35000 & 877000 & 24500 \\
$\mathrm{~J} 1833-0827$ & $\mathrm{G} 24.7-0.6$ & 3.4 & 8.2 & $5 \times 10^{-6}$ & $2.5 \times 10^{-5}$ & 70000 & 147000 & 18500 \\
$\mathrm{~J} 1915+1009$ & $\mathrm{G} 45.7-0.4$ & 0.7 & 4.9 & $2 \times 10^{-5}$ & $1 \times 10^{-4}$ & 200000 & 420000 & 47000 \\
$\mathrm{~J} 1832-0827$ & $\mathrm{G} 22.7-0.2$ & 0.62 & 6.8 & $3 \times 10^{-5}$ & $3 \times 10^{-4}$ & 140000 & 161000 & 4000 \\
$\mathrm{~J} 1835-1106$ & $\mathrm{G} 18.9-1.1$ & 0.64 & 4 & $5 \times 10^{-5}$ & $1 \times 10^{-4}$ & 40000 & 128000 & 6000 \\
$\mathrm{~J} 1832-0827$ & $\mathrm{G} 23.3-0.3$ & 1.5 & 3.3 & $3 \times 10^{-5}$ & $1.2 \times 10^{-4}$ & 180000 & 161000 & 20500 \\
$\mathrm{~J} 1835-1106$ & $\mathrm{G} 22.7-0.2$ & 2.1 & 2.4 & $1 \times 10^{-4}$ & $5 \times 10^{-5}$ & 70000 & 128000 & 4000 \\
$\mathrm{~J} 1833-0827$ & $\mathrm{G} 23.3-0.3$ & 2.4 & 1.2 & $2 \times 10^{-4}$ & $9.3 \times 10^{-6}$ & 150000 & 147000 & 20500 \\
\hline
\end{tabular}

Table 1: $\rho_{\min }$ - the minimum separation found among the simulated trajectories; $\rho_{0, \min }$ - the minimum separation found for random encounters; $P(5), P_{0}(5)$ - corresponding probabilities of encounters at distances $\rho \leq 5 \mathrm{pc} ; T(5)$ - the median epoch that characterizes the approaches to $\rho \leq 5 \mathrm{pc} ; \tau_{c h}$ - characteristic age of the pulsar; $t_{S N R}-$ age of the SNR.

Pulsar J1833-0827 and SNR G24.7-0.6 show a difference of 5 times between $P(\rho \leq 5 \mathrm{pc})$ and $P_{0}(\rho \leq 5 \mathrm{pc})$. The age range for this SNR is $2500-65000$ years, and the epoch of approach is 60000-80000 years. This might be a case of an old SNR associated with a pulsar whose actual age may be around 60000 years (see Figures 3 and 4).

The pair of J1915+1009 and G45.7-0.4 also shows a probability difference of 5 times. However, the epoch of approach here is 200000 years, and while this is marginally possible, it seems not very likely that the SNR may actually be that old.

J1832-0827 and G22.7-0.2 show a probability difference of 10 times. The epoch of approach, 140000 years, and the characteristic age of the pulsar, 161000 years, are also in good agreement. The presumed age of the SNR, on the other hand, is only a few thousand years, which would make it difficult for these objects to be associated unless the SNR is actually much older. This is unlikely given its small diameter.

The probability $P(\rho \leq 5 \mathrm{pc})$ for $\mathrm{J} 1835-1106$ and G18.9-1.1 is twice as high as $P_{0}(\rho \leq 5 \mathrm{pc})$. However, to declare a possible association, we need at least a difference of 3 times. In this case we can only say that this pair slightly stands out kinematically among the others.

Another unlikely, but not completely ruled out association is that of J1832-0827 and G23.30.3. Despite the probability difference of 4 , the age of the SNR is smaller than required for such an association.

The remaining association candidates, J1835-1106 and G22.7-0.2, and J1833-0827 and G23.30.3 , have proved to be unlikely associations, despite fitting the initial selection criteria. In both cases, $P_{0}(\rho \leq 5 \mathrm{pc})$ is greater than $P(\rho \leq 5 \mathrm{pc})$, which means that any approach between the objects is a coincidence, despite the relatively good agreement of $\tau_{c h}$ and the epoch of approach, as well as their proximity in space. 


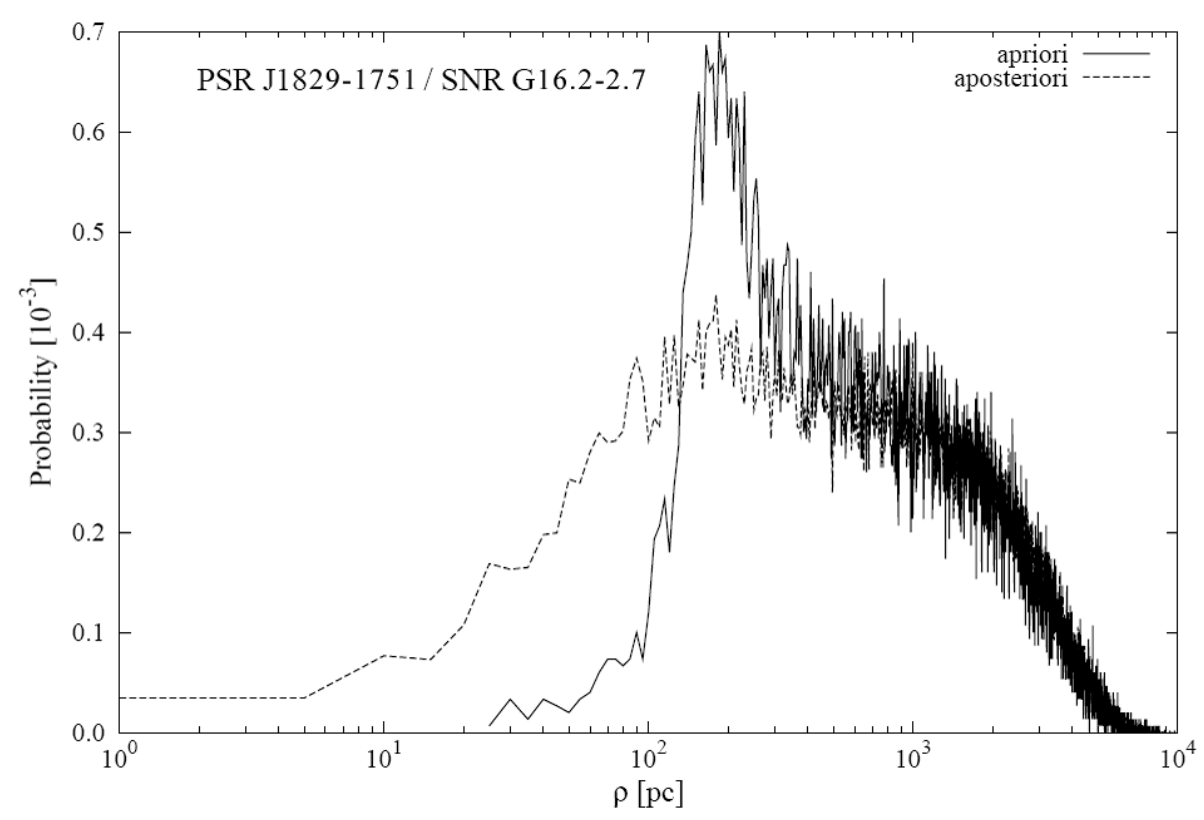

Figure 1: J1829-1751 and G16.2-2.7: Here and in Figure 2: The relation of encounter probability and distance $\rho$ between the objects. The dashed lines represent $P(\rho)$ - the probability of an encounter between the objects calculated during our simulations. The solid lines represent $P_{0}(\rho)$ - the probability of random encounters.

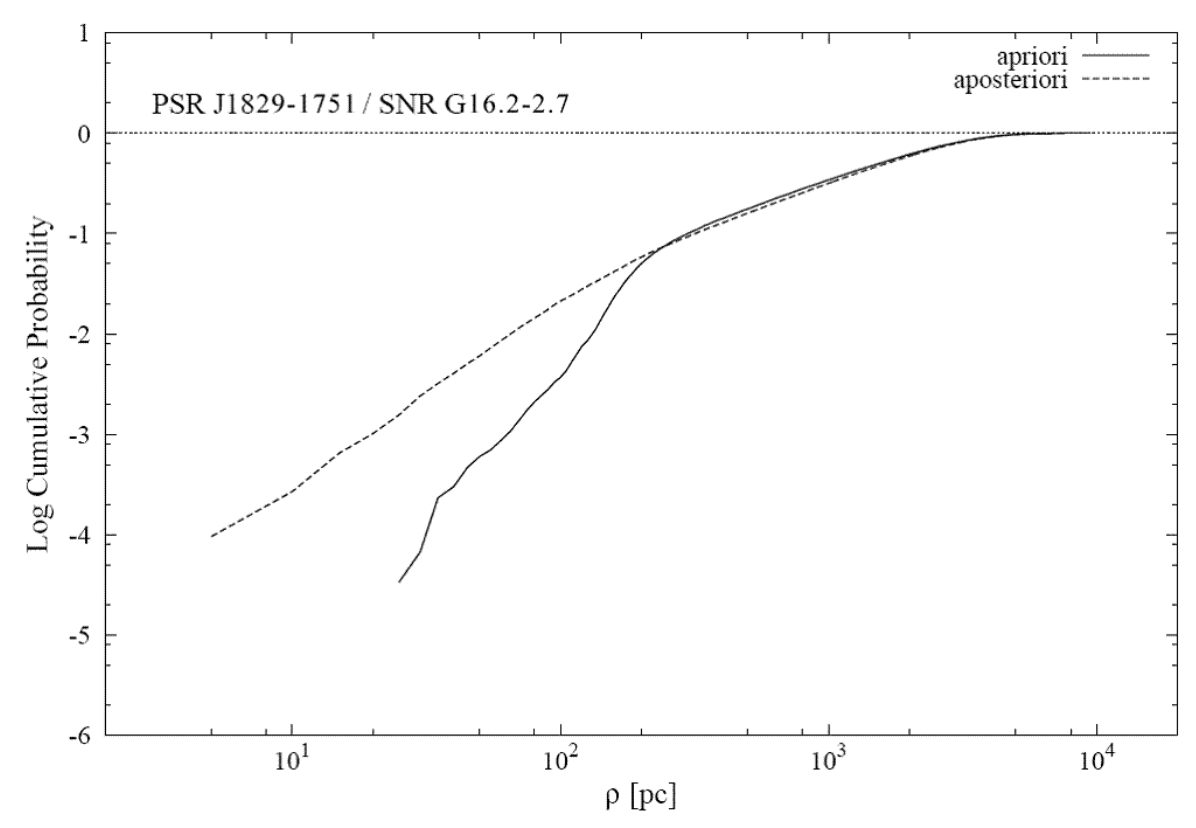

Figure 2: J1829-1751 and G16.2-2.7 


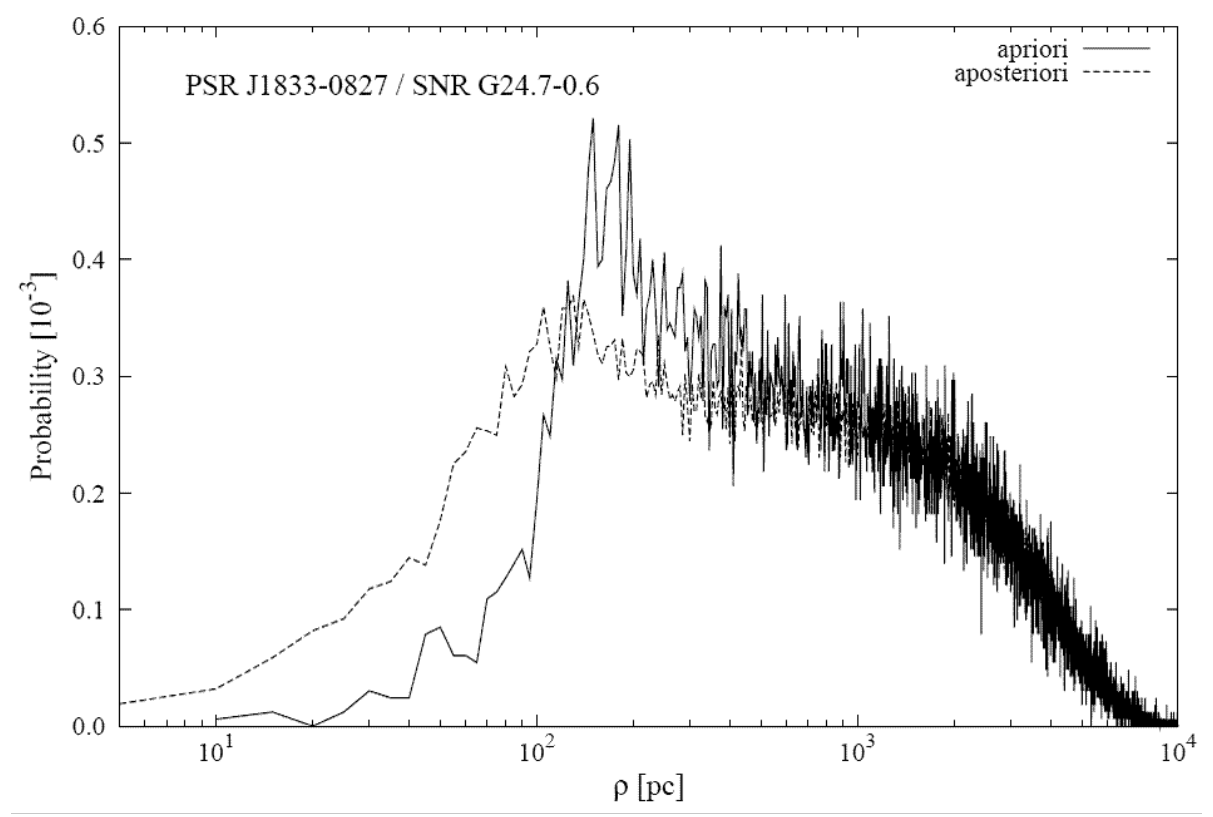

Figure 3: J1833-0827 and G24.7-0.6: Here and in Figure 4: The relation of encounter probability and distance $\rho$ between the objects. The dashed lines represent $P(\rho)$ - the probability of an encounter between the objects calculated during our simulations. The solid lines represent $P_{0}(\rho)$ - the probability of random encounters.

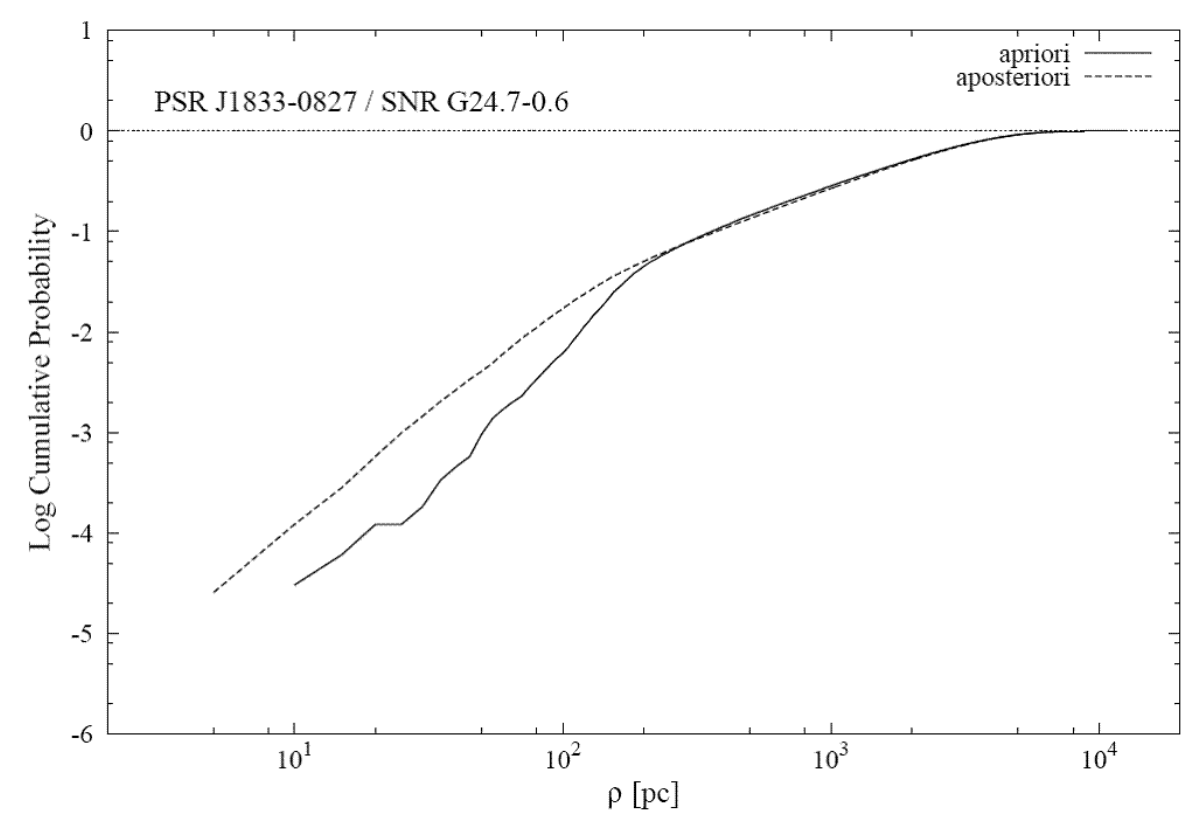

Figure 4: J1833-0827 and G24.7-0.6 


\section{References}

[1] G. Hobbs, D.R. Lorimer, et al., A statistical study of 233 pulsar proper motions, MNRAS 360, 974 (2005).

[2] E. van der Swaluw, A. Achterberg, et al., Interaction of high-velocity pulsars with supernova remnant shells, A\&A 397, 913 (2003).

[3] E.G. Chmyreva, G.M. Beskin, et al., Search for pairs of isolated radio pulsars - components in disrupted binary systems, AstL 36, 116 (2010).

[4] R.N. Manchester, G.B. Hobbs, et al., The Australia Telescope National Facility pulsar catalogue, AJ 129, 1993 (2005).

[5] D.A. Green, A revised Galactic supernova remnant catalogue, BASI 37, 45 (2009).

[6] J.-W. Xu, X.-Z. Zhang, et al., Statistics of Galactic supernova remnants, ChJAA 5, 165 (2005). 